\title{
An Exact Axisymmetric Solution in Anisotropic Plasticity
}

\author{
Yaroslav Erisov ${ }^{1,2}$, Sergei Surudin ${ }^{1,2}$, Fedor Grechnikov ${ }^{2, *}$ and Elena Lyamina ${ }^{1,3}$ \\ 1 Metal Forming Department, Samara National Research University, 34 Moskovskoye Shosse, \\ 443086 Samara, Russia; yaroslav.erisov@mail.ru (Y.E.); surudin.sv@ssau.ru (S.S.); lyamina@inbox.ru (E.L.) \\ 2 Samara Federal Research Center of the Russian Academy of Sciences, 3A Studencheskiy Pereulok, \\ 443001 Samara, Russia \\ 3 Ishlinsky Institute for Problems in Mechanics, 101-1 Prospect Vernadskogo, 119526 Moscow, Russia \\ * Correspondence: gretch@ssau.ru
}

check for

updates

Citation: Erisov, Y.; Surudin, S.; Grechnikov, F.; Lyamina, E. An Exact Axisymmetric Solution in Anisotropic Plasticity. Symmetry 2021, 13, 825. https://doi.org/10.3390/sym13050825

Academic Editor: Danny Arrigo

Received: 19 March 2021

Accepted: 1 May 2021

Published: 8 May 2021

Publisher's Note: MDPI stays neutral with regard to jurisdictional claims in published maps and institutional affiliations.

Copyright: (C) 2021 by the authors. Licensee MDPI, Basel, Switzerland. This article is an open access article distributed under the terms and conditions of the Creative Commons Attribution (CC BY) license (https:/ / creativecommons.org/licenses/by/ $4.0 /)$.

\begin{abstract}
A hollow cylinder of incompressible material obeying Hill's orthotropic quadratic yield criterion and its associated flow rule is contracted on a rigid cylinder inserted in its hole. Friction occurs at the contact surface between the hollow and solid cylinders. An axisymmetric boundary value problem for the flow of the material is formulated and solved, and the solution is in closed form. A numerical technique is only necessary for evaluating ordinary integrals. The solution may exhibit singular behavior in the vicinity of the friction surface. The exact asymptotic representation of the solution shows that some strain rate components and the plastic work rate approach infinity in the friction surface's vicinity. The effect of plastic anisotropy on the solution's behavior is discussed.
\end{abstract}

Keywords: plastic anisotropy; friction; singularity; exact solution

\section{Introduction}

One can rarely find an analytical solution to an axisymmetric problem in plasticity. Such solutions exist for the one-dimensional classical problems, such as expanding tubes and spherical shells [1]. A solution for the flow of rigid perfectly plastic material through an infinite conical channel has been presented in [2]. This solution is valid for an arbitrary isotropic pressure-independent yield criterion. Several papers have generalized the solution [2]. In particular, a solution for the flow of rigid/linear-hardening materials has been given in [3]. This solution has been further generalized on an arbitrary nonlinear strainhardening law in [4]. Paper [5] has dealt with the radial flow of rigid/linear-hardening materials between two conical walls. The solution [2] has been used in [6,7] for constructing a solution for the flow of multilayered material. The same approach in conjunction with the solutions given in [4,5] has been adopted in [6] to include strain hardening into consideration. The radial flow of viscoplastic solids has been studied in [8], where solutions for other rate-dependent materials have been discussed. Solutions with non-zero circumferential velocity have been derived in $[9,10]$. In both papers, the material response is rigid perfectly plastic. Paper [11] has generalized the solution [2] on the double shearing model of ideal granular material. The double shearing model is described in this paper as well. It is worthy of note that of the solutions above, only the rigid perfectly plastic solutions are exact. The other solutions involve more approximations or have more limitations than the solution [2].

Another series of exact solutions has started from the solution given in [12]. Considered is a rigid/perfectly plastic hollow cylinder with a rigid solid cylinder inserted in its hole. The rigid/perfectly plastic cylinder is subject to uniform contraction over its outer surface. The constitutive equations comprise Tresca's yield criterion and its associated flow rule. In [13] the solution [12] has been extended to the rigid/perfectly plastic material model based on the yield criterion proposed in [14]. Papers [15] and [16] have generalized the solution [12] on a model of viscous material and the double shearing model, respectively. 
The importance of analytical solutions is at least twofold. Firstly, such solutions can be used as benchmark problems for verifying numerical codes, which a necessary step before using such codes $[17,18]$. Secondly, analytical solutions are useful for understanding the qualitative behavior of general solutions. Of particular interest for rigid/plastic models is the maximum friction surfaces where the velocity field may be singular [19]. This singularity causes difficulty with numerical solutions. In particular, finite element solutions based on typical shape functions do not converge [20,21].

One of the present paper's objectives is to extend the solution [12] to a model of anisotropic plasticity. It is known that even mild plastic anisotropy may significantly affect some features of isotropic solutions [22]. An example of such an effect has been presented, for example, in [23]. It is assumed that the material obeys Hill's quadratic yield criterion and its associate flow rule [1]. This model is widely used until now [24-27]. It is known that plane strain solutions for this model may be singular [28]. This singularity is associated with envelopes of characteristics. The equations for axisymmetric flow are not hyperbolic. However, isolated characteristic surfaces in ideal plasticity may exist even if the equations are not hyperbolic [29]. Another objective of the present paper is to demonstrate it for the model adopted using a particular solution.

Many theoretical and experimental studies have been devoted to the failure of continuous fiber-reinforced ductile matrix composites [12,30-37]. All these works can be divided into three groups, namely (i) matrix damage [30-32]; (ii) matrix-fiber interface fracture (debonding) [33-36]; and (iii) fiber breakage [12,36,37]. An applied aspect of the solution found is that it can be used for predicting the brittle fracture of fibers in such composites using the approach proposed in [12]. The third objective of the present paper is to combine this approach and the solution found.

\section{Statement of the Problem}

A rigid solid cylinder is inserted into the hole of a rigid/plastic hollow cylinder (Figure 1). The radius of the rigid cylinder and the radius of the hollow cylinder's hole is denoted by $a_{0}$, and the outer radius of the hollow cylinder by $b_{0}$. The length of both the cylinders is $2 \mathrm{~L}$. The hollow cylinder is subject to uniform contraction over its outer surface. Cylindrical coordinates $(r, \theta, z)$ are taken, with the $z$-axis coinciding with the axis of symmetry of each cylinder. The plane $z=0$ is a plane of symmetry of the boundary value problem. The solution is independent of $\theta$. The boundary conditions are the same as those in [12]. In particular, the boundary conditions at $r=a_{0}$ and $r=b_{0}$ are satisfied exactly, except for the condition on the radial stress. The exact boundary conditions at $z=0$ and $z=L$ are replaced with integral boundary conditions. Since $z=0$ is the plane of symmetry for the flow, it is sufficient to find the solution in the region $0 \leq z \leq L$.

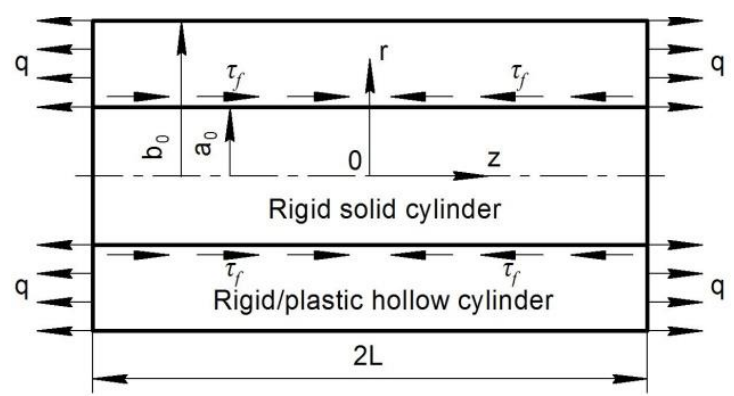

Figure 1. Configuration and coordinate system.

The non-zero components of the stress tensor in the cylindrical coordinates are denoted by $\sigma_{r r}, \sigma_{\theta \theta}, \sigma_{z z}$, and $\sigma_{r z}$; and the non-zero components of the velocity vector by $u_{r}$ and $u_{z}$. Since the inner cylinder is rigid,

$$
u_{r}=0 \text {, }
$$


for $r=a_{0}$. The other velocity boundary condition that is exactly satisfied is

$$
u_{r}=-U,
$$

for $r=b_{0}$. Here $U>0$. The exact velocity boundary condition at the plane of symmetry is replaced with

$$
\int_{a_{0}}^{b_{0}} u_{z} r d r=0,
$$

for $z=0$. The stress boundary conditions that are exactly satisfied are

$$
\sigma_{r z}=0,
$$

for $r=b_{0}$ and Prandtl's friction law at $r=a_{0}$. This law depends on the constitutive equations and will be formulated below. The integral stress boundary condition proposed in [12] reads

$$
\left.\int_{0}^{L} \sigma_{r r}\right|_{r=b_{0}} d z=0 .
$$

At the end of the hollow cylinder, an axial stress is required to deform it according to the boundary conditions above. Once the boundary value problem has been solved, the average value of this stress is calculated as

$$
q=\left.\frac{2}{\left(b_{0}^{2}-a_{0}^{2}\right)} \int_{a_{0}}^{b_{0}} \sigma_{z z}\right|_{z=L} r d r .
$$

This stress is involved in the method for predicting the brittle fracture of fibers in composites proposed in [12].

The material of the hollow cylinder is plastically orthotropic. The principal axes of anisotropy coincide with coordinate curves of the cylindrical coordinate system. The elastic portion of strain is neglected. The constitutive equations comprise the yield criterion proposed in [1] and its associated flow rule. In the case under consideration, the yield criterion reads

$$
F\left(\sigma_{\theta \theta}-\sigma_{z z}\right)^{2}+G\left(\sigma_{z z}-\sigma_{r r}\right)^{2}+H\left(\sigma_{r r}-\sigma_{\theta \theta}\right)^{2}+2 M \sigma_{r z}^{2}=1 .
$$

Here

$$
2 F=\frac{1}{\Theta^{2}}+\frac{1}{Z^{2}}-\frac{1}{R^{2}}, \quad 2 G=\frac{1}{Z^{2}}+\frac{1}{R^{2}}-\frac{1}{\Theta^{2}}, \quad 2 H=\frac{1}{R^{2}}+\frac{1}{\Theta^{2}}-\frac{1}{Z^{2}} .
$$

where $R$ is the tensile yield stress in the radial direction, $\Theta$ is the tensile yield stress in the circumferential direction, $Z$ is the tensile yield stress in the axial direction, and $1 / \sqrt{2 M}$ is the shear yield stress with respect to the $r$-, $z$-axes. All of them are supposed to be constant. The flow rule is

$$
\begin{gathered}
\xi_{r r}=\lambda\left[H\left(\sigma_{r r}-\sigma_{\theta \theta}\right)+G\left(\sigma_{r r}-\sigma_{z z}\right)\right], \\
\xi_{\theta \theta}=\lambda\left[F\left(\sigma_{\theta \theta}-\sigma_{z z}\right)+H\left(\sigma_{\theta \theta}-\sigma_{r r}\right)\right], \\
\xi_{z z}=\lambda\left[G\left(\sigma_{z z}-\sigma_{r r}\right)+F\left(\sigma_{z z}-\sigma_{\theta \theta}\right)\right], \\
\xi_{r z}=\lambda M \sigma_{r z} .
\end{gathered}
$$

Here $\lambda$ is a non-negative multiplier.

Prandtl's friction law for isotropic materials postulates that the friction stress $\tau_{f}$ is a fraction of the shear yield stress. Therefore, it is reasonable in the case under consideration to represent this law as

$$
\sigma_{r z}=\tau_{f}=\frac{m}{\sqrt{2 M}}
$$


for $r=a_{0}$. Here $m$ is the friction factor, $0 \leq m \leq 1$.

In what follows, it is convenient to use the nondimensional quantities:

$$
\rho=\frac{r}{b_{0}}, \zeta=\frac{z}{b_{0}} \text { and } a=\frac{a_{0}}{b_{0}} .
$$

\section{Analytic Solution}

The solution provided in [12] suggests that one looks for velocity solutions of the form

$$
\frac{u_{r}}{U}=-\eta(\rho) \text { and } \frac{u_{z}}{U}=A \zeta+\mu(\rho) .
$$

Here $\eta(\rho)$ and $\mu(\rho)$ are arbitrary functions only of $\rho$, and $A$ is constant. Equation (8) results in the equation of incompressibility $\xi_{\rho \rho}+\xi_{\theta \theta}+\xi_{z z}=0$. In terms of the velocity components, this equation becomes $\partial u_{r} / \partial r+u_{r} / r+\partial u_{z} / \partial z=0$. Or, using Equation (10),

$$
\frac{\partial u_{r}}{\partial \rho}+\frac{u_{r}}{\rho}+\frac{\partial u_{z}}{\partial \zeta}=0
$$

Substituting Equation (11) into Equation (12) one gets

$$
\frac{d \eta}{d \rho}+\frac{\eta}{\rho}=A
$$

This equation can be immediately integrated to give

$$
\eta=\frac{A \rho}{2}+\frac{A_{0}}{\rho} .
$$

Here $A_{0}$ is constant. Equations (1), (2), (10), (11), and (14) combine to give

$$
A_{0}=-\frac{a^{2}}{1-a^{2}} \text { and } A=\frac{2}{1-a^{2}} .
$$
leads to

Substituting Equation (15) into Equation (14) and the resulting equation into (11)

$$
\frac{u_{r}}{U}=-\frac{1}{\left(1-a^{2}\right)}\left(\rho-\frac{a^{2}}{\rho}\right) \text { and } \frac{u_{z}}{U}=\frac{2 \zeta}{\left(1-a^{2}\right)}+\mu(\rho) .
$$

Then, using Equation (10), one can find the non-zero components of the strain rate tensor referred to the cylindrical coordinates as

$$
\begin{aligned}
& \frac{b_{0}}{U} \xi_{r r}=\frac{b_{0}}{U} \frac{\partial u_{r}}{\partial r}=-\frac{1}{\left(1-a^{2}\right)}\left(\rho+\frac{a^{2}}{\rho^{2}}\right), \frac{b_{0} \xi_{\theta \theta}}{U}=\frac{b_{0}}{U} \frac{u_{r}}{r}=-\frac{1}{\left(1-a^{2}\right)}\left(1-\frac{a^{2}}{\rho^{2}}\right), \\
& \frac{b_{0} \tilde{z}_{z z}}{U}=\frac{b_{0}}{U} \frac{\partial u_{z}}{\partial z}=\frac{2}{\left(1-a^{2}\right)}, \frac{b_{0} \tilde{\xi}_{r z}}{U}=\frac{b_{0}}{2 U} \frac{\partial u_{z}}{\partial r}=\frac{1}{2} \frac{d \mu}{d \rho} .
\end{aligned}
$$

In a generic meridian plane, the slope $\varphi$ of the principal axes of strain rate with respect to the $r$-axis is given by

$$
\tan 2 \varphi=\frac{2 \xi_{r z}}{\xi_{r r}-\xi_{z z}}=\left(\frac{\partial u_{r}}{\partial z}+\frac{\partial u_{z}}{\partial r}\right)\left(\frac{\partial u_{r}}{\partial r}-\frac{\partial u_{z}}{\partial z}\right)^{-1}
$$

Equations (17) and (18) combine to give

$$
\tan 2 \varphi=\frac{\rho^{2}\left(a^{2}-1\right)}{\left(a^{2}+3 \rho^{2}\right)} \frac{d \mu}{d \rho} .
$$


The slope $\varphi$ can also be expressed in terms of the stress components using Equations (8) and (18). As a result,

$$
\tan 2 \varphi=\frac{2 M \sigma_{r z}}{H\left(\sigma_{r r}-\sigma_{\theta \theta}\right)+2 G\left(\sigma_{r r}-\sigma_{z z}\right)-F\left(\sigma_{z z}-\sigma_{\theta \theta}\right)} .
$$

It is convenient to introduce the new stress variables $p_{1}, p_{2}$, and $p_{3}$ as

$$
p_{1}=H\left(\sigma_{r r}-\sigma_{\theta \theta}\right), p_{2}=F\left(\sigma_{\theta \theta}-\sigma_{z z}\right), p_{3}=G\left(\sigma_{z z}-\sigma_{r r}\right) .
$$

It is evident that

$$
\frac{p_{1}}{H}+\frac{p_{2}}{F}+\frac{p_{3}}{G}=0
$$

Equations (20) and (21) combine to give

$$
\frac{2 M \sigma_{r z}}{p_{1}-2 p_{3}+p_{2}}=\tan 2 \varphi \text {. }
$$

It follows from Equations (8) and (21) that $\xi_{r r}\left(p_{2}-p_{1}\right)=\xi_{\theta \theta}\left(p_{1}-p_{3}\right)$ or $\left(p_{2}-p_{1}\right) \partial u_{r} / \partial r=\left(p_{1}-p_{3}\right) u_{r} / r$. Using Equations (10) and (16), one transforms the latter equation to

$$
\frac{p_{1}-p_{3}}{p_{2}-p_{1}}=t
$$

One can solve Equations (22) and (24) for $p_{3}$ and $p_{2}$ to get

$$
p_{2}=p_{1} w \text { and } p_{3}=p_{1}[1+t(1-w)] .
$$

In Equations (24) and (25), $t$ and $w$ are functions of $\rho$ defined as

$$
t=\frac{\rho^{2}+a^{2}}{\rho^{2}-a^{2}}, w=\left[\frac{1}{H}+\frac{(1+t)}{G}\right]\left(\frac{t}{G}-\frac{1}{F}\right)^{-1} .
$$

Equations (23) and (25) combine to give

$$
2 M \sigma_{r z}=p_{1}(w-1)(1+2 t) \tan 2 \varphi
$$

Substituting Equation (21) into Equation (7) and using Equations (25) and (27), one arrives at the following equation for $p_{1}$

$$
p_{1}^{2}=\left\{\frac{w^{2}}{F}+\frac{[1+t(1-w)]^{2}}{G}+\frac{1}{H}+\frac{\tan ^{2} 2 \varphi(w-1)^{2}(1+2 t)^{2}}{2 M}\right\}^{-1}
$$

Assuming that the components of the deviatoric stress tensor are independent of $z$, the equilibrium equations reduce to

$$
\frac{\partial \sigma}{\partial \rho}+\frac{d \tau_{r r}}{d \rho}+\frac{\tau_{r r}-\tau_{\theta \theta}}{\rho}=0 \text { and } \frac{d \sigma_{r z}}{d \rho}+\frac{\partial \sigma}{\partial \zeta}+\frac{\sigma_{r z}}{\rho}=0
$$

Here $\sigma$ is the hydrostatic stress, $\tau_{r r}=\sigma_{r r}-\sigma$, and $\tau_{\theta \theta}=\sigma_{\theta \theta}-\sigma$. The third normal component of the deviatoric stress tensor is $\tau_{z z}=\sigma_{z z}-\sigma$. The Equations in (29) are compatible if

$$
\sqrt{2 M} \sigma=2 B \zeta+\sqrt{2 M} \sigma_{0}(\rho)
$$

where $B$ is constant and $\sigma_{0}(\rho)$ is a function of $\rho$. Substituting Equation (30) into Equation (29), one gets

$$
\frac{d \sigma_{0}}{d \rho}+\frac{d \tau_{r r}}{d \rho}+\frac{\tau_{r r}-\tau_{\theta \theta}}{\rho}=0 \text { and } \frac{d \sigma_{r z}}{d \rho}+\frac{\sigma_{r z}}{\rho}=-\frac{\sqrt{2} B}{\sqrt{M}} .
$$


The second equation in Equation (31) can be immediately integrated to give

$$
\sqrt{2 M} \sigma_{r z}=\frac{C}{\rho}-B \rho,
$$

where $C$ is constant. The boundary conditions in Equations (4) and (9) serve for determining $B$ and $C$. As a result, using Equation (10),

$$
C=B=\frac{m a}{1-a^{2}} .
$$

Then, Equation (32) becomes

$$
\sqrt{2 M} \sigma_{r z}=\frac{m a}{\left(1-a^{2}\right)}\left(\frac{1}{\rho}-\rho\right) .
$$

Equations (27) and (34) combine to give

$$
p_{1}=\frac{m \sqrt{2 M} a\left(1-\rho^{2}\right)}{\rho\left(1-a^{2}\right)(w-1)(1+2 t) \tan 2 \varphi} .
$$

Eliminating $p_{1}$ between Equations (28) and (35), one arrives at the following equation for $\tan 2 \varphi$

$$
\tan ^{2} 2 \varphi=\frac{2 M m^{2} a^{2}\left(1-\rho^{2}\right)^{2}}{(w-1)^{2}(1+2 t)^{2}\left[\rho^{2}\left(1-a^{2}\right)^{2}-m^{2} a^{2}\left(1-\rho^{2}\right)^{2}\right]}\left\{\frac{w^{2}}{F}+\frac{[1+t(1-w)]^{2}}{G}+\frac{1}{H}\right\} .
$$

Substituting $\tan 2 \varphi$ back into Equation (35) leads to

$$
p_{1}= \pm \frac{\sqrt{\rho^{2}\left(1-a^{2}\right)^{2}-m^{2} a^{2}\left(1-\rho^{2}\right)^{2}}}{\rho\left(1-a^{2}\right) \sqrt{\frac{w^{2}}{F}+\frac{[1+t(1-w)]^{2}}{G}+\frac{1}{H}}} .
$$

It is seen from Equation (26) that $t>0, t \rightarrow \infty$ as $\rho \rightarrow a$, and $d t / d \rho<0$ for all $\rho$. Moreover, $w \rightarrow 1$ as $t \rightarrow \infty$ (or $\rho \rightarrow a$ ) and $d w / d t<0$ for all $t$. Summarizing the relations above, one can conclude that the direction of the friction stress (Figure 1) demands that $d \mu / d \rho>0$. Then, Equations (19) and (36) combine to give

$$
w \geq 1 \text { and } t>0 .
$$

$$
\frac{d \mu}{d \rho}=\frac{\sqrt{2 M} m a\left(1-\rho^{2}\right)\left(a^{2}+3 \rho^{2}\right)}{\left(1-a^{2}\right)(w-1)(1+2 t) \rho^{2} \sqrt{\rho^{2}\left(1-a^{2}\right)^{2}-m^{2} a^{2}\left(1-\rho^{2}\right)^{2}}} \sqrt{\frac{w^{2}}{F}+\frac{[1+t(1-w)]^{2}}{G}+\frac{1}{H}} .
$$

Here, Equation (38) has been taken into account. It follows from Equation (39) that

$$
\mu=\frac{\sqrt{2 M} m a}{\left(1-a^{2}\right)} \int_{1}^{\rho} \frac{\left(1-\chi^{2}\right)\left(a^{2}+3 \chi^{2}\right)}{(w-1)(1+2 t) \chi^{2} \sqrt{\chi^{2}\left(1-a^{2}\right)^{2}-m^{2} a^{2}\left(1-\chi^{2}\right)^{2}}} \sqrt{\frac{w^{2}}{F}+\frac{[1+t(1-w)]^{2}}{G}+\frac{1}{H}} d \chi+\mu_{0} .
$$

Here, $\mu_{0}$ is constant. Its value is found applying Equation (3). Using Equations (10), (11) and (40), one gets

$$
\mu_{0}=-\frac{2 \sqrt{2 M} m a}{\left(1-a^{2}\right)^{2}} \int_{a}^{1} \rho \int_{1}^{\rho} \frac{\left(1-\chi^{2}\right)\left(a^{2}+3 \chi^{2}\right)}{(w-1)(1+2 t) \chi^{2} \sqrt{\chi^{2}\left(1-a^{2}\right)^{2}-m^{2} a^{2}\left(1-\chi^{2}\right)^{2}}} \sqrt{\frac{w^{2}}{F}+\frac{[1+t(1-w)]^{2}}{G}+\frac{1}{H}} d \chi d \rho .
$$

The integrals in Equations (40) and (41) should be evaluated numerically. 
Equation (21) is equivalent to

$$
p_{1}=H\left(\tau_{r r}-\tau_{\theta \theta}\right), p_{2}=F\left(\tau_{\theta \theta}-\tau_{z z}\right), p_{3}=G\left(\tau_{z z}-\tau_{r r}\right) .
$$

These equations can be solved for the components of the deviatoric stress tensor. As a result,

$$
\tau_{r r}=\frac{1}{3}\left(\frac{2 p_{1}}{H}+\frac{p_{2}}{F}\right), \tau_{\theta \theta}=\frac{1}{3}\left(\frac{p_{2}}{F}-\frac{p_{1}}{H}\right), \tau_{z z}=-\frac{1}{3}\left(\frac{2 p_{2}}{F}+\frac{p_{1}}{H}\right) .
$$

Substituting Equation (43) into the first equation in Equation (31), one finds

$$
\frac{d\left(\sigma_{0}+\tau_{r r}\right)}{d \rho}=-\frac{p_{1}}{\rho H}
$$

Equations (19), (35), and the inequality $d \mu / d \rho>0$ demands that $p_{1}<0$. Then, the lower sign should be chosen in Equation (37). Using this equation, one can rewrite Equation (44) as

$$
\frac{d\left(\sigma_{0}+\tau_{r r}\right)}{d \rho}=\frac{\sqrt{\rho^{2}\left(1-a^{2}\right)^{2}-m^{2} a^{2}\left(1-\rho^{2}\right)^{2}}}{H \rho^{2}\left(1-a^{2}\right) \sqrt{\frac{w^{2}}{F}+\frac{[1+t(1-w)]^{2}}{G}+\frac{1}{H}}} .
$$

Integrating and using Equation (43) gives

$$
\sigma_{0}+\tau_{r r}=\frac{1}{H\left(1-a^{2}\right)} \int_{1}^{\rho} \frac{\sqrt{\chi^{2}\left(1-a^{2}\right)^{2}-m^{2} a^{2}\left(1-\chi^{2}\right)^{2}}}{\chi^{2} \sqrt{\frac{w^{2}}{F}+\frac{[1+t(1-w)]^{2}}{G}+\frac{1}{H}}} d \chi+D .
$$

Equation (5) serves for determining $D$. The radial stress is found from Equations (30), (33) and (46) as

$$
\sigma_{r r}=\frac{2 m a \zeta}{\sqrt{2 M}\left(1-a^{2}\right)}+\sigma_{0}+\tau_{r r}=\frac{2 m a \zeta}{\sqrt{2 M}\left(1-a^{2}\right)}+\frac{1}{H\left(1-a^{2}\right)} \int_{1}^{\rho} \frac{\sqrt{\chi^{2}\left(1-a^{2}\right)^{2}-m^{2} a^{2}\left(1-\chi^{2}\right)^{2}}}{\chi^{2} \sqrt{\frac{w^{2}}{F}+\frac{[1+t(1-w)]^{2}}{G}+\frac{1}{H}}} d \chi+D .
$$

Substituting Equation (47) into Equation (5) and using Equation (10) gives

$$
D=-\frac{m a}{\sqrt{2 M}\left(1-a^{2}\right)}\left(\frac{L}{b_{0}}\right)
$$

This equation completes the solution.

Summarizing the solution above, one finds the velocity field from Equations (16), (40) and (41). Equations (34), (47), and (48) supply the shear and radial stresses. The other normal stresses follow from Equations (30) and (33), and the equations $\sigma_{\theta \theta}=\sigma+\tau_{\theta \theta}$ and $\sigma_{z z}=\sigma+\tau_{z z}$. The deviatoric stress components involved in these equations are determined from Equations (25), (37) and (43). Having found the axial stress, one calculates the value of $q$ from Equation (6).

The plastic work rate $W$ can be calculated as [1]:

$$
W=\bar{\sigma} \bar{\xi},
$$

where $\bar{\sigma}$ and $\bar{\xi}$ are the equivalent stress and strain rate, respectively, which are given by

$$
\bar{\sigma}=\sqrt{\frac{3}{2(F+G+H)}} \sqrt{F\left(\sigma_{\theta \theta}-\sigma_{z z}\right)^{2}+G\left(\sigma_{z z}-\sigma_{r r}\right)^{2}+H\left(\sigma_{r r}-\sigma_{\theta \theta}\right)^{2}+2 M \sigma_{r z}^{2}}
$$




$$
\bar{\xi}=\sqrt{\frac{2(F+G+H)}{3}} \sqrt{F\left(\frac{G \xi_{\theta \theta}-H \xi_{z z}}{F G+G H+H F}\right)^{2}+G\left(\frac{H \xi_{z z}-F \xi_{r r}}{F G+G H+H F}\right)^{2}+H\left(\frac{F \xi_{r r}-G \xi_{\theta \theta}}{F G+G H+H F}\right)^{2}+\frac{2 \xi_{r z}^{2}}{M}} .
$$

\section{Asymptotic Analysis}

Many rigid/plastic solutions are singular in the vicinity of maximum friction surfaces $[19,28]$. In the case under consideration, the maximum friction surface is $\rho=a$ if $m=1$. Equation (39) at $m=1$ becomes

$$
\frac{d \mu}{d \rho}=\frac{\sqrt{2 M} a\left(1-\rho^{2}\right)\left(a^{2}+3 \rho^{2}\right)}{\left(1-a^{2}\right)(w-1)(1+2 t) \rho^{2} \sqrt{\rho^{2}\left(1-a^{2}\right)^{2}-a^{2}\left(1-\rho^{2}\right)^{2}}} \sqrt{\frac{w^{2}}{F}+\frac{[1+t(1-w)]^{2}}{G}+\frac{1}{H}} .
$$

It is seen from Equation (26) that $t \rightarrow \infty$ as $\rho \rightarrow a$. However,

$$
\lim _{\rho \rightarrow a}[t(\rho)(1-w(\rho))]=-\frac{G}{F}-\frac{G}{H}-1
$$

Therefore, the function $\rho^{2}\left(1-a^{2}\right)^{2}-a^{2}\left(1-\rho^{2}\right)^{2}$ controls the behavior of the righthand side of Equation (52) as $\rho \rightarrow a$. Since

$$
\rho^{2}\left(1-a^{2}\right)^{2}-a^{2}\left(1-\rho^{2}\right)^{2}=2 a\left(1-a^{4}\right)(\rho-a)+o[(\rho-a)]
$$

as $\rho \rightarrow a$, it is evident from Equation (52) that

$$
\frac{d \mu}{d \rho}=O\left(\frac{1}{\sqrt{\rho-a}}\right)
$$

as $\rho \rightarrow a$. Then, it follows from Equation (11) that the shear strain rate approaches infinity in the vicinity of the maximum friction surface. In particular,

$$
\xi_{r z}=O\left(\frac{1}{\sqrt{\rho-a}}\right)
$$

as $\rho \rightarrow a$. The normal strain rates referred to the cylindrical coordinate system are not singular.

Using Equations (53) and (54), one can rewrite Equation (45) as

$$
\frac{d\left(\sigma_{0}+\tau_{r r}\right)}{d \rho}=O[\sqrt{\rho-a}],
$$

as $\rho \rightarrow a$. Integrating

$$
\sigma_{0}+\tau_{r r}=O\left[(\rho-a)^{3 / 2}\right]+D,
$$

as $\rho \rightarrow a$. It follows from Equations (25) and (37) in which the lower sign is chosen, (53), and (54) that

$$
\begin{gathered}
p_{1}=-\sqrt{\frac{2\left(1+a^{2}\right)}{a\left(1-a^{2}\right)}}\left[\frac{1}{F}+\frac{1}{H}+\frac{1}{G}\left(\frac{G}{F}+\frac{G}{H}+1\right)^{2}\right]^{-1 / 2} \sqrt{\rho-a}+o(\sqrt{\rho-a}), \\
p_{2}=-\sqrt{\frac{2\left(1+a^{2}\right)}{a\left(1-a^{2}\right)}}\left[\frac{1}{F}+\frac{1}{H}+\frac{1}{G}\left(\frac{G}{F}+\frac{G}{H}+1\right)^{2}\right]^{-1 / 2} \sqrt{\rho-a}+o(\sqrt{\rho-a}), \\
p_{3}=\sqrt{\frac{2\left(1+a^{2}\right)}{a\left(1-a^{2}\right)}}\left(\frac{G}{F}+\frac{G}{H}\right)\left[\frac{1}{F}+\frac{1}{H}+\frac{1}{G}\left(\frac{G}{F}+\frac{G}{H}+1\right)^{2}\right]^{-1 / 2} \sqrt{\rho-a}+o(\sqrt{\rho-a}),
\end{gathered}
$$

as $\rho \rightarrow a$. If $F \neq H$, Equations (43) and (59) combine to give

$$
\tau_{r r}=O(\sqrt{\rho-a}), \tau_{\theta \theta}=O(\sqrt{\rho-a}) \text {, and } \tau_{z z}=O(\sqrt{\rho-a}),
$$


as $\rho \rightarrow a$. If $F=H$, the second equation in Equation (60) transforms to

$$
\tau_{\theta \theta}=o(\sqrt{\rho-a}),
$$

as $\rho \rightarrow a$. Comparing Equations (58) and (60), one concludes that

$$
\sigma_{0}=O(\sqrt{\rho-a})+D,
$$

as $\rho \rightarrow a$. At any $\zeta$, it follows from Equations (30) and (62) that

$$
\sigma=O(\sqrt{\rho-a})+D_{1}
$$

as $\rho \rightarrow a$. Equations (58), (60), and (63) show that the absolute values of the derivatives $\partial \sigma_{\theta \theta} / \partial \rho$ and $\partial \sigma_{z z} / \partial \rho$ approach infinity as $\rho \rightarrow a$ but the absolute value of the derivative $\partial \sigma_{r r} / \partial \rho$ does not.

The asymptotic Expansions (56), (60), (61) and (63) show that traditional finite elements are not capable of solving this problem. It has been already demonstrated in [21] for an isotropic model that calculation does not converge. These asymptotic expansions can be used in conjunction with the extended finite element method [38].

Equations (51) and (56) show that the equivalent strain rate approaches infinity near the bi-material interface. This strain rate controls the evolution of many material properties. Therefore, Equations (51) and (56) predict a high gradient of such properties near the bimaterial interface. This phenomenon has been known for a long time (see, for example, [39]). In particular, the generation of microstructure in the vicinity of bi-material interfaces has been studied in [40,41]. Nevertheless, the asymptotic expansions found cannot be directly used in conjunction with conventional evolution equations for material properties because $\bar{\xi} \rightarrow \infty$ as $\rho \rightarrow a$. An approach to overcome this difficulty for isotropic materials has been proposed in [42]. Equation (56) suggests that a similar approach can be developed for anisotropic materials.

\section{Numerical Example}

The numerical example provided in this section evaluates the effect of the hollow cylinder's material anisotropy on the stress and strain fields. It was assumed that the hollow cylinder is made from aluminum alloy AA5086. Due to various parameters of the manufacturing process, the same material may exhibit different anisotropic properties characterized by the symmetry of mechanical properties with respect to the principal axes of anisotropy [43-45]. The following types of anisotropy were considered: isotropic, transversely isotropic (mechanical properties are identical in any direction in the $z \theta$-coordinate surface but differ from the properties in the other coordinate surfaces) and orthotropic materials. The AA5086 alloy's mechanical properties involved in yield criterion (7) are available in the literature [46]. The mechanical properties responsible for plastic anisotropy are summarized in Table 1.

Table 1. Input data for all the cases considered.

\begin{tabular}{cccccc}
\hline Material & $\mathbf{Z}, \mathbf{M P a}$ & $\begin{array}{c}\mathbf{F}, \mathbf{1 0}^{-\mathbf{6}} \\
\mathbf{M P a}^{-\mathbf{2}}\end{array}$ & $\begin{array}{c}\mathbf{G}, \mathbf{1 0}^{-\mathbf{6}} \\
\mathbf{M P a}^{-\mathbf{2}}\end{array}$ & $\begin{array}{c}\mathbf{H}, \mathbf{1 0}^{-\mathbf{6}} \\
\mathbf{M P a}^{-\mathbf{2}}\end{array}$ & $\begin{array}{c}\mathbf{M}, \mathbf{1 0}^{-\mathbf{6}} \\
\mathbf{M P a}^{-\mathbf{2}}\end{array}$ \\
\hline Isotropic & 191 & 13.7 & 13.7 & 13.7 & 41.1 \\
$\begin{array}{c}\text { Transversely } \\
\text { isotropic }\end{array}$ & 191 & 9.0 & 18.4 & 18.4 & 36.4 \\
Orthotropic & 191 & 10.8 & 16.6 & 20.8 & 43.7 \\
\hline
\end{tabular}

The solution provided in Section 3 has been applied to calculate the distribution of the velocities, strain rates, stresses, and plastic work rate at $m=1$. It has also been assumed that $a^{2}=1 / 2$ and $L / b_{0}=10$. The first equation in Equation (16) supplies the radial distribution of the radial velocity at any value of $a$. This simple formula does not require a 
graphical illustration. No material properties affect the radial velocity. The second equation in Equation (16) shows that the function $\mu(\rho)$ completely controls the influence of plastic anisotropy on the axial velocity. Figure 2 illustrates this function. Using this Figure, one can visualize the radial distribution of the axial velocity with ease by adding the first term on the second equation's right-hand side in Equation (16). This term is constant at any value of $\zeta$. It is seen from Figure 2 that the influence of plastic anisotropy on the axial velocity is not significant. However, one can see an interesting qualitative feature of the solution that the magnitude of this velocity in the vicinity of the friction surface is largest for transversely isotropic material, even though this material model is intermediate with respect to the other two models. Another qualitative feature of the solution is that the magnitude of the axial velocity in the cylinder of transversely isotropic material is the largest in the vicinity of the friction surface but is the smallest at the outside radius, as compared to the other two models. The curves in Figure 2 are in qualitative agreement with Equation (55).

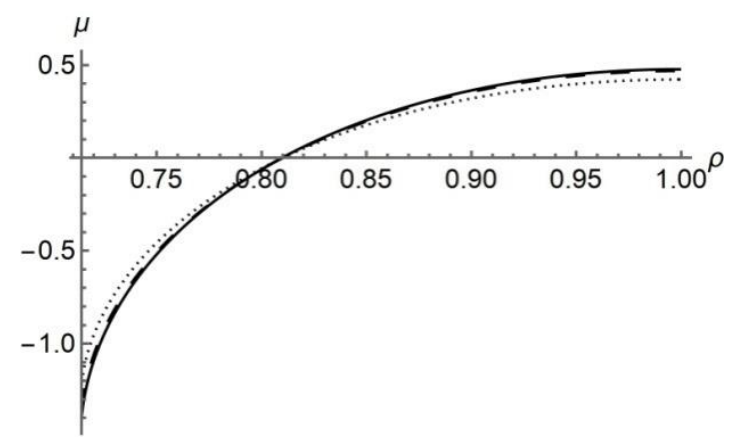

Figure 2. Illustration of the function $\mu(\rho)$ : — - isotropic material; - - - - orthotropic material; .... transversely isotropic material.

The strain rate tensor's normal components are given by the simple formulae presented in Equation (17). Therefore, no graphical illustration of these components is required. The last equation in Equation (17) shows that the function $\mu(\rho)$ completely controls the influence of plastic anisotropy on the shear strain rate component. The variation of this component with the dimensionless radius is depicted in Figure 3. The effect of plastic anisotropy is not significant. As in the case of the axial velocity, the curve corresponding to transversely isotropic material does not lie between the curves corresponding to the isotropic and orthotropic materials. The magnitude of the shear strain rate approaches infinity in the vicinity of the friction surface in accordance with Equation (56).

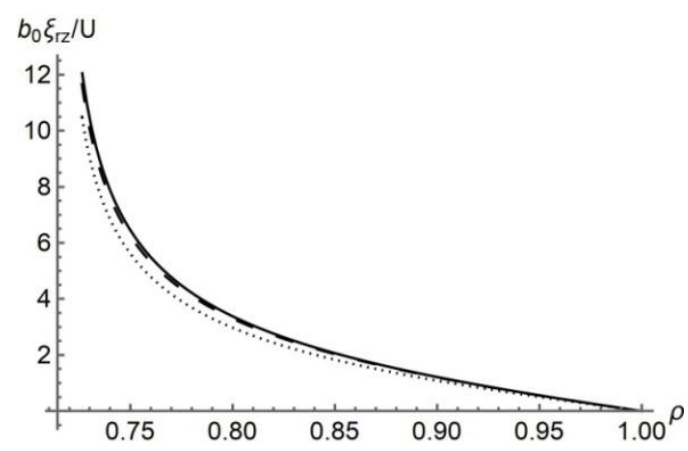

Figure 3. Radial distribution of the shear strain rate: - - isotropic material; - - - - orthotropic material; $\cdots$ - transversely isotropic material.

The simple formula in Equation (34) gives the solution for the shear stress. The effect of plastic anisotropy vanishes if this stress is normalized by $\sqrt{M}$. Therefore, no graphical illustration of this stress is required. The most significant influence of plastic anisotropy is observed on $\tau_{r r}, \tau_{\theta \theta}$, and $\sigma$ (Figure 4). The change of the type of anisotropy affects 
these distributions quantitively. In particular, the isotropic model results in the largest absolute value of $\tau_{r r}$, the smallest absolute value of $\tau_{\theta \theta}$, and the intermediate value of $\sigma$. The transversely isotropic model results in the smallest absolute value of $\tau_{r r}$, the largest absolute value of $\tau_{\theta \theta}$, and the largest absolute value of $\sigma$. The orthotropic model results in the intermediate absolute values of both $\tau_{r r}$ and $\tau_{\theta \theta}$, and the smallest absolute value of $\sigma$. The effect of the type of plastic anisotropy on the deviatoric stress $\tau_{z z}$ is negligible. The difference in the value of $\sigma$ between the orthotropic and transversely isotropic models is about $10 \%$. This result is important for predicting the brittle fracture of fibers in composites using the method proposed in [12]. It is seen from Figure 4 that the tangents to all the curves corresponding $\tau_{r r}, \tau_{z z}$, and $\sigma$ tend to a vertical line in the vicinity of the inner radius. This behavior of the curves is in accordance with Equations (60) and (63). The curves corresponding to $\tau_{\theta \theta}$ reveal the same feature for the orthotropic and transversely isotropic models but not for the isotropic model. The latter agrees with Equation (61) since $F=H=G$ in the case.
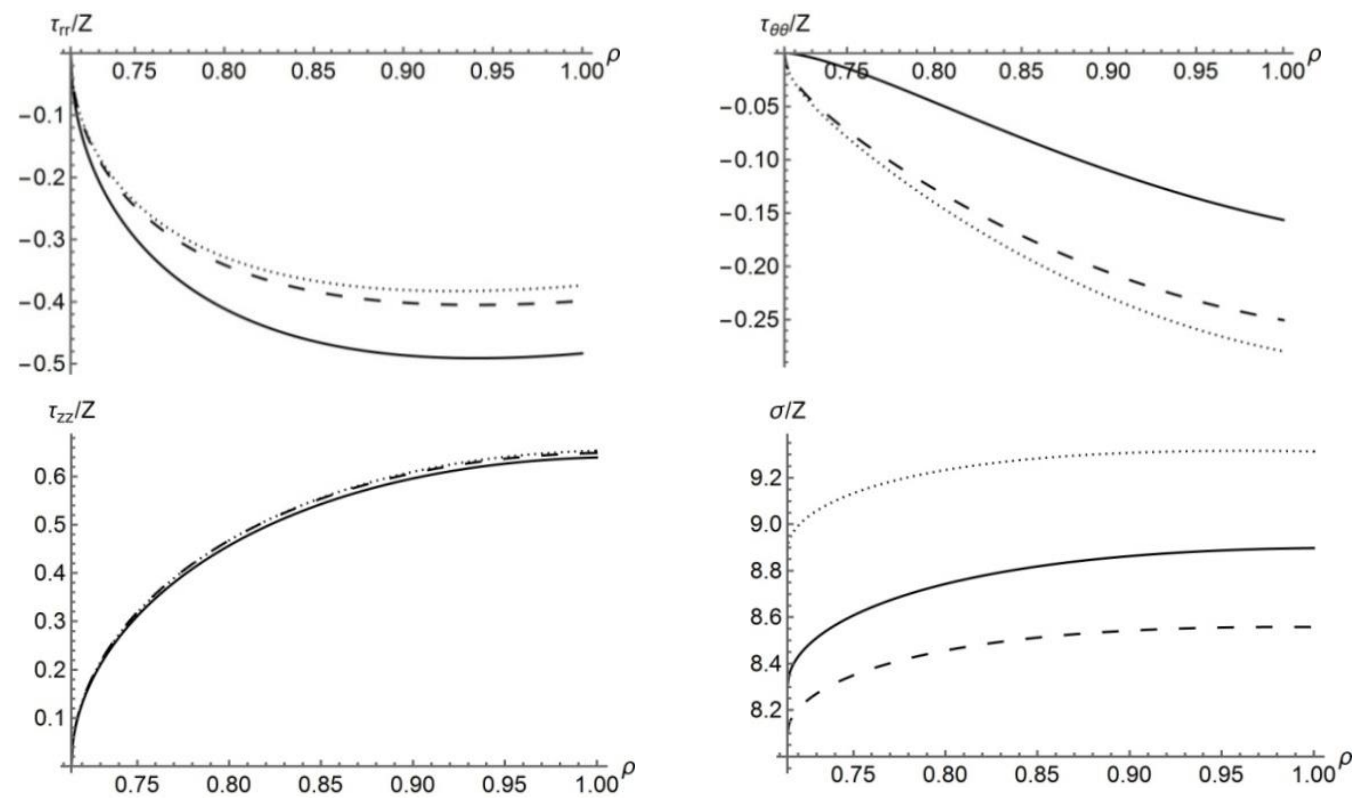

Figure 4. Radial distribution of the deviatoric and hydrostatic stresses: - - isotropic material; - - - orthotropic material; $\cdots$ - - transversely isotropic material.

The radial distribution of the plastic work rate calculated using Equations (49)-(51) is depicted in Figure 5. Since the stresses and normal strain rates are bounded, it is seen from these equations and Equation (56) that $W=O(1 / \sqrt{\rho-a})$ as $\rho \rightarrow a$. This asymptotic representation of the solution is visible in Figure 5.

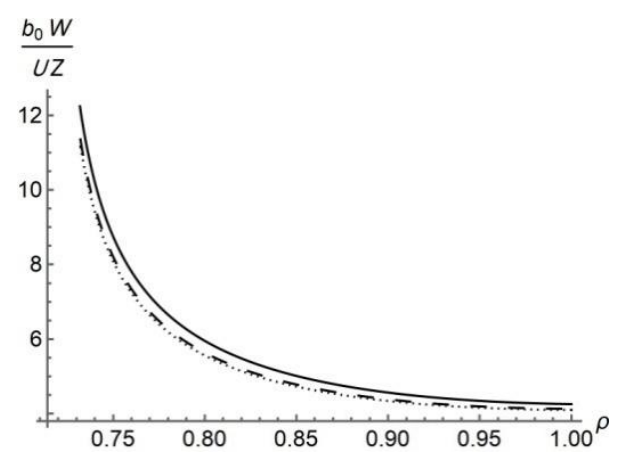

Figure 5. Radial distribution of the plastic work rate: - - isotropic material; - - - - orthotropic material; .... - transversely isotropic material. 


\section{Prediction of the Brittle Fracture of Fibers in Composites}

The solution above can be used for predicting the failure of composite sheets made of ductile matrix and brittle fibers subject to tension in the direction of fibers using the approach suggested in [12]. According to this approach, the failure occurs by plastic flow of the matrix if $L<L_{c}$ and by brittle fracture of the fiber if $L>L_{c}$. The critical length $L_{c}$ should be found from the solution. The criterion for finding this length is that both failure mechanisms occur simultaneously. Equation (6) at $L=L_{c}$ supplies the tensile load $q_{c}$ at which the fiber breaks. The parameters from Section 5 have been used in the calculation. In addition, the mean tensile stress at which the fiber breaks, $T$, is required. Its magnitude has been varied in the range typical for the fibrous composites [47].

The change of the type of anisotropy affects the ultimate tensile strength quantitively (Figure 6). In particular, the orthotropic model results in the largest values of $q_{c}$ on the range of $T$, the smallest values of $q_{c}$ correspond to the transversely isotropic model; the isotropic model results in the intermediate values. The lower the difference between the ultimate stress of the fiber and the yield stress of the matrix, the greater the effect of the plastic properties anisotropy on the brittle fracture of the fiber.

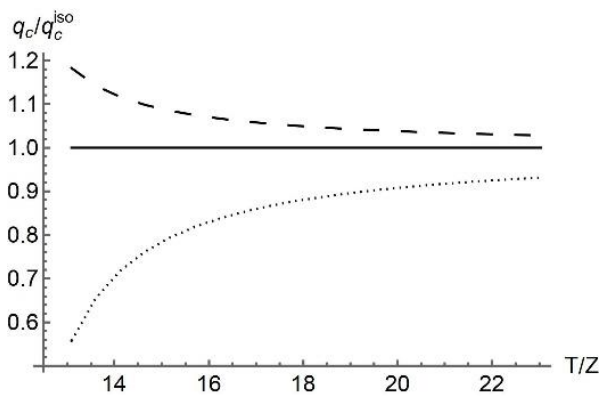

Figure 6. Dependence of the tensile load at which the fiber breaks on its ultimate stress: - - isotropic material; - - - - orthotropic material; .... - transversely isotropic material.

\section{Conclusions}

An exact axisymmetric solution has been found for the stress and velocity fields in a hollow rigid/plastic anisotropic cylinder contracted a solid rigid cylinder. Three types of anisotropy have been adopted for obtaining quantitative results: orthotropic material, transversely isotropic material, and isotropic material. The following conclusions have been reached from this theoretical analysis:

1. The type of anisotropy has a negligible effect on kinematics variables and the plastic work rate. This feature is attributed to the boundary conditions that specify the radial velocity at both the inner and outer radii of the rigid/plastic cylinder and the equation of incompressibility valid for all the models adopted.

2. The type of anisotropy has a significant effect on the radial and circumferential deviatoric stress and hydrostatic stress. The type of anisotropy has a negligible effect on the axial deviatoric stress. A consequence of such a solution's behavior is that the type of anisotropy has a significant effect on the axial stress involved in Equation (6).

3. If the friction factor is equal to unity, then the solution becomes singular in the vicinity of the inner radius of the rigid/plastic cylinder. In particular, the shear strain rate and plastic work rate approach infinity near this radius according to an inverse square root rule. This behavior may cause difficulties with solving other problems using numerical methods.

4. The solution found can be used in conjunction with the approach proposed in [12] for predicting the brittle fracture of fibers in composites.

Author Contributions: Methodology, F.G.; formal analysis, E.L.; investigation, Y.E. and S.S.; writingoriginal draft preparation, Y.E.; writing-review and editing, S.S. All authors have read and agreed to the published version of the manuscript. 
Funding: This research was funded by the Russian Science Foundation, grant number 20-79-10340.

Institutional Review Board Statement: Not applicable.

Informed Consent Statement: Not applicable.

Conflicts of Interest: The authors declare no conflict of interest.

\section{Nomenclature}

$\begin{array}{ll}a_{0} & \text { Radius of rigid cylinder [mm] } \\ b_{0} & \text { Outer radius of hollow cylinder [mm] } \\ 2 L & \text { Length of both cylinders [mm] } \\ L_{c} & \text { Critical fiber length [mm] } \\ \rho, \zeta, a & \text { Nondimensional quantities } \\ (r, \theta, z) & \text { Cylindrical coordinates } \\ \sigma_{r r}, \sigma_{\theta \theta}, \sigma_{z z}, \sigma_{r z} & \text { Components of the stress tensor in the cylindrical coordinates [MPa] } \\ \tau_{f} & \text { Friction stress [MPa] } \\ \xi_{r r}, \xi_{\theta \theta}, \xi_{z z}, \xi_{r z} & \left.\text { Components of the strain rate tensor in the cylindrical coordinates [s }{ }^{-1}\right] \\ u_{r}, u_{z} & \text { Components of the velocity vector [mm/s] } \\ U & \text { Radial velocity at te outer radius [mm/s] } \\ q & \text { Average value of axial stress [MPa] } \\ q_{c} & \text { Tensile load at which the fiber breaks in composite [MPa] } \\ \sigma & \text { Hydrostatic stress [MPa] } \\ \tau_{r r}, \tau_{\theta \theta}, \tau_{z z} & \text { Components of the deviatoric stress tensor [MPa] } \\ F, G, H, M & \text { Hill's coefficients [MPa }{ }^{-2} \text { ] } \\ R, \Theta, Z & \text { Tensile yield stresses in the radial, circumferential and axial directions [MPa] } \\ \lambda & \text { Non-negative multiplier } \\ m & \text { Friction factor } \\ \eta, \mu, \mathrm{t}, \mathrm{w}, \sigma_{0} & \text { Functions of } \\ \varphi & \text { Slope of the principal axes of strain rate with respect to the r-axis } \\ A, A_{0}, B, C, \mu_{0}, D & \text { Constants } \\ p_{1}, p_{2}, p_{3} & \text { Stress variables [MPa }{ }^{-1} \text { ] } \\ W & \text { Plastic work rate [MPa/s] } \\ \bar{\sigma} & \text { Equivalent stress [MPa] } \\ \bar{\xi} & \text { Equivalent strain rate [s }{ }^{-1} \text { ] } \\ T & \text { Mean tensile stress at which the fiber breaks [MPa] }\end{array}$

\section{References}

1. Hill, R. The Mathematical Theory of Plasticity; Clarendon Press: Oxford, UK, 1950.

2. Shield, R.T. Plastic flow in a converging conical channel. J. Mech. Phys. Solids 1955, 3, 246-258. [CrossRef]

3. Durban, D. Axially Symmetric Radial Flow of Rigid/Linear-Hardening Materials. ASME J. Appl. Mech. 1979, 46, 322-328. [CrossRef]

4. Durban, D. Radial flow simulation of drawing and extrusion of rigid/hardening materials. Int. J. Mech. Sci. 1983, 25, 27-39. [CrossRef]

5. $\quad$ Durban, D. Drawing of Tubes. ASME J. Appl. Mech. 1980, 47, 736-740. [CrossRef]

6. Durban, D. Drawing and extrusion of composite sheets, wires and tubes. Int. J. Solids Struct. 1984, 20, 649-666. [CrossRef]

7. Danishevs'kyy, V.V.; Guillet, A.; Pareige, P.; Weichert, D. An analytical approach to the plastic flow of a bimetallic monofilamentary wire through a conical die. Mech. Mater. 2008, 40, 284-292. [CrossRef]

8. Durban, D. On generalized radial flow patterns of viscoplastic solids with some applications. Int. J. Mech. Sci 1986, 28, 97-110. [CrossRef]

9. Brovman, M.J. Steady forming processes of plastic materials with their rotation. Int. J. Mech. Sci. 1987, 29, 483-489. [CrossRef]

10. Durban, D.; Davidi, G.; Lior, D. Plastic Forming Processes Through Rotating Conical Dies. ASME J. Appl. Mech. 2001, 68, 894-902. [CrossRef]

11. Spencer, A.J.M. Deformation of ideal granular materials. In Mechanics of Solids; Hopkins, H.G., Sewell, M.J., Eds.; Pergamon Press: Oxford, UK, 1982; pp. 607-652.

12. Spencer, A.J.M. A theory of the failure of ductile materials reinforced by elastic fibres. Int. J. Mech. Sci. 1965, 7, 197-209. [CrossRef]

13. Alexandrov, S.; Erisov, Y.; Grechnikov, F. Effect of the Yield Criterion of Matrix on the Brittle Fracture of Fibres in Uniaxial Tension of Composites. Adv. Mater. Sci. Eng. 2016, 2016. [CrossRef]

14. Hosford, W.F. A Generalized Isotropic Yield Criterion. ASME J. Appl. Mech. 1972, 39, 607-609. [CrossRef] 
15. Alexandrov, S.; Jeng, Y.-R. A Generalization of Prandtl's and Spencer's Solutions on Axisymmetric Viscous Flow. Arch. Appl. Mech. 2011, 81, 437-449. [CrossRef]

16. Aleksandrov, S.E.; Goldstein, R.V. Generalization of the Prandtl Solution to the Case of Axisymmetric Deformation of Materials Obeying the Double Shear Model. Mech. Solids 2012, 47, 654-664. [CrossRef]

17. Roberts, S.M.; Hall, F.R.; Van Bael, A.; Hartley, P.; Pillinger, I.; Sturgess, C.E.N.; Van Houtte, P.; Aernoudt, E. Benchmark tests for 3-D, elasto-plastic, finite-element codes for the modelling of metal forming processes. J. Mater. Process. Technol. 1992, 34, 61-68. [CrossRef]

18. Abali, B.E.; Reich, F.A. Verification of deforming polarized structure computation by using a closed-form solution. Cont. Mech. Therm. 2020, 32, 693-708. [CrossRef]

19. Alexandrov, S.; Richmond, O. Singular Plastic Flow Fields Near Surfaces of Maximum Friction Stress. Int. J. Non-Linear Mech. 2001, 36, 1-11. [CrossRef]

20. Chen, J.-S.; Pan, C.; Roque, C.M.O.L.; Wang, H.-P. A Lagrangian reproducing kernel particle method for metal forming analysis. Comput. Mech. 1998, 22, 289-307. [CrossRef]

21. Facchinetti, M.; Mishuris, G. Analysis of the maximum friction condition for green body forming in an ANSYS environment. J. Eur. Ceram. Soc. 2016, 36, 2295-2302. [CrossRef]

22. Prime, M. Amplified effect of mild plastic anisotropy on residual stress and strain anisotropy. Int. J. Solids Struct. 2017, 118-119, 70-77. [CrossRef]

23. Rynkovskaya, M.; Alexandrov, S.; Lang, L. A Theory of Autofrettage for Open-Ended, Polar Orthotropic Cylinders. Symmetry 2019, 11, 280. [CrossRef]

24. Xu, M.; Zhao, Y.; Zhou, B.; He, X.; Zhou, C. Limit Load Solutions of the Orthotropic Thick-Walled Pipe Subjected to Internal Pressure, Bending Moment and Torsion Moment. In Proceedings of the ASME 2019 Pressure Vessels E Piping Conference; American Society of Mechanical Engineers: New York, NY, USA, 2019; Volume 3. [CrossRef]

25. Qin, F.; Zhang, L.; Chen, G.; Broeckmann, C. Lower bound limit and shakedown analysis of orthotropic material. Math. Mech. Solids 2020, 25, 2037-2049. [CrossRef]

26. Wang, C.; Ramakrishnan, K.R.; Shankar, K.; Morozov, E.; Wang, H.; Fien, A. Homogenized shell element-based modeling of low-velocity impact response of stainless-steel wire mesh. Mech. Adv. Mater. Struct. 2020. [CrossRef]

27. Kořínek, M.; Halama, R.; Fojtík, F.; Pagáč, M.; Krček, J.; Krzikalla, D.; Kocich, R.; Kunčická, L. Monotonic tension-torsion experiments and FE modeling on notched specimens produced by SLM technology from SS316l. Materials 2021, 14, 33. [CrossRef] [PubMed]

28. Alexandrov, S.; Jeng, Y.-R. Singular Rigid/Plastic Solutions in Anisotropic Plasticity Under Plane Strain Conditions. Cont. Mech. Therm. 2013, 25, 685-689. [CrossRef]

29. Craggs, J.W. Characteristic Surfaces in Ideal Plasticity in Three Dimensions. Quart. J. Mech. Appl. Math. 1954, 7, 35-39. [CrossRef]

30. Voyiadjis, G.Z.; Thiagarajan, G. Micro and macro anisotropic cyclic damage-plasticity models for MMCs. Int. J. Eng. Sci. 1997, 35, 467-484. [CrossRef]

31. Son, C.-Y.; Kim, G.S.; Lee, S.-B.; Lee, S.-K.; Kim, H.S.; Huh, H.; Lee, S. Correlation of microstructure with mechanical properties of Zr-based amorphous matrix composite reinforced with tungsten continuous fibers and ductile dendrites. Metall. Mater. Trans. A Phys. Metall. Mater. Sci. 2012, 43, 4088-4096. [CrossRef]

32. Arefi, A.; Van der Meer, F.P.; Forouzan, M.R.; Silani, M.; Salimi, M. Micromechanical evaluation of failure models for unidirectional fiber-reinforced composites. J. Compos. Mater. 2020, 54, 791-800. [CrossRef]

33. Eldridge, J.I.; Brindley, P.K. Investigation of interfacial shear strength in a SiC fibre/Ti-24Al-11Nb composite by a fibre push-out technique. J. Mater. Sci. Lett. 1989, 8, 1451-1454. [CrossRef]

34. Allen, D.H.; Jones, R.H.; Boyd, J.G. Micromechanical analysis of a continuous fiber metal matrix composite including the effects of matrix viscoplasticity and evolving damage. J. Mech. Phys. Solids 1994, 42, 505-529. [CrossRef]

35. Pupurs, A.; Varna, J. Steady-state energy release rate for fiber/matrix interface debond growth in unidirectional composites. Int. J. Damage Mech. 2017, 26, 560-587. [CrossRef]

36. Pupurs, A. Fiber failure and debonding in composite materials. In Modeling Damage, Fatigue and Failure of Composite Materials; Woodhead Publishing: Cambridge, UK, 2015; pp. 175-196. [CrossRef]

37. $\mathrm{Wu}, \mathrm{Y} . ; \mathrm{Ju}, \mathrm{J} . \mathrm{W}$. Elastoplastic damage micromechanics for continuous fiber-reinforced ductile matrix composites with progressive fiber breakage. Int. J. Damage Mech. 2017, 26, 3-27. [CrossRef]

38. Fries, T.-P.; Belytschko, T. The extended/generalized finite element method: An overview of the method and its applications. Int. J. Numer. Methods Eng. 2010, 84, 253-304. [CrossRef]

39. Griffiths, B.J. Mechanisms of White Layer Generation with Reference to Machining and Deformation Processes. ASME J. Tribol. 1987, 109, 525-530. [CrossRef]

40. Sasaki, T.T.; Morris, R.A.; Thompson, G.B.; Syarif, Y.; Fox, D. Formation of ultra-fine copper grains in copper-clad aluminum wire. Scripta Mater. 2010, 63, 488-491. [CrossRef]

41. Thirumurugan, M.; Rao, S.A.; Kumaran, S.; Rao, T.S. Improved ductility in ZM21 magnesium-aluminiummacrocomposite produced by co-extrusion. J. Mater. Process. Technol. 2011, 211, 1637-1642. [CrossRef]

42. Goldstein, R.V.; Alexandrov, S.E. An Approach to Prediction of Microstructure Formation Near Friction Surfaces at Large Plastic Strains. Phys. Mesomech. 2015, 18, 223-227. [CrossRef] 
43. Suwas, S.; Ray, R.K. Crystallographic Texture of Materials; Springer: London, UK, 2014. [CrossRef]

44. Ivanov, Y.F.; Gromov, V.E.; Konovalov, S.V.; Zagulyaev, D.V.; Petrikova, E.A.; Semin, A.P. Modification of structure and surface properties of hypoeutectic silumin by intense pulse electron beams. Prog. Phys. Met. 2018, 19, 195-222. [CrossRef]

45. Zhang, C.; Lv, P.; Xia, H.; Yang, Z.; Konovalov, S.; Chen, X.; Guan, Q. The microstructure and properties of nanostructured Cr-Al alloying layer fabricated by high-current pulsed electron beam. Vacuum 2019, 167, 263-270. [CrossRef]

46. Zhang, S.; Leotoing, L.; Guines, D.; Thuillier, S.; Zang, S. Calibration of anisotropic yield criterion with conventional tests or biaxial test. Int. J. Mech. Sci. 2014, 85, 142-151. [CrossRef]

47. Vinson, J.R.; Sierakowski, R.L. Introduction to Composite Materials. In The Behavior of Structures Composed of Composite Materials; Springer: Dordrecht, The Netherlands, 1987; pp. 1-27. [CrossRef] 